\title{
PLANOS DE SAÚDE: UMA ANÁLISE DOS CUSTOS ASSISTENCIAIS E SEUS COMPONENTES
}

HEALTH INSURANCE PLANS: AN ANALYSIS OF CARE COSTS AND THEIR COMPONENTS

\section{RESUMO}

A partir da motivação de contribuir para a investigação dos custos em saúde, o objetivo deste trabalho é analisar a evolução dos custos de assistência médica dos planos de saúde no Brasil, considerando a distinção marcante entre as categorias de contratação. Para esse fim, utilizaram-se as informações divulgadas pela Agência Nacional de Saúde Suplementar (ANS), sendo estimados índices de variação com base na metodologia de índices de valor e seus dois componentes, preço (custo médio por evento) e quantidade (frequência de utilização). Dentre os resultados encontrados, destaca-se que os índices de custo médio por evento apresentaram variação mais intensa do que os índices de quantidade, fato que aponta no sentido da importância do aprofundamento da pesquisa sobre a inflação em saúde.

\section{Rodrigo Mendes Leal}

Economista do Banco Nacional de Desenvolvimento Econômico e Social - RJ, Brasil e Doutorando em Políticas Públicas, Estratégia e Desenvolvimento pelo Instituto de Economia, Universidade Federal do Rio de Janeiro - RJ, Brasil rmendesleal@gmail.com

\section{João Boaventura Branco de Matos}

Especialista em Regulação da Agência Nacional de Saúde - RJ, Brasil; Professor da Escola Superior de Propaganda e Marketing - RJ, Brasil e Doutorando pelo Instituto de Medicina Social, Universidade do Estado do Rio de Janeiro - RJ, Brasil jmatos@espm.br

Recebido em 31.03.2008. Aprovado em 08.07.2009

Avaliado pelo sistema double blind review

Editores Científicos: Organizadores do Fórum

ABSTRACT The objective of this work is to contribute to the investigation into health costs, by analyzing the evolution of the cost of medical care in health plans in Brazil, considering the marked difference between the categories of contract available. To do so, the information published by the National Supplementary Health Agency (AINS) is used. The variation indices are estimated, using the value indices methodology and its two components, price (average cost per event) and quantity (use frequency). What stands out in the results is that the average cost indices per event have varied more that the quantity indices, a fact which indicates how important it is to research health inflation in more depth.

PALAVRAS-CHAVE Planos de saúde, custos em saúde, inflação, administração da saúde, preços em saúde. KEYWORDS Health plans, health costs, inflation, health administration, health prices. 


\section{INTRODUÇÃO}

Os planos de saúde constituem no Brasil uma importante via de atendimento ao cidadão, tendo em vista que atualmente cerca da quarta parte da população está associada a algum tipo de plano, conforme informações da Agência Nacional de Saúde Suplementar (doravante ANS) (ANS, 2007). A regulação desse setor se consolidou a partir de 1998, com a Lei n 9.656/1998, e especialmente a partir de 2000, com a criação da ANS (SANTOS, 2007). Atualmente a regulação tem evoluído no sentido de consolidar os avanços realizados na área da regulação das empresas, fortalecer os aspectos técnico-assistenciais e estimular a concorrência entre as operadoras. Essa tendência de reforço dos aspectos técnicos da regulação e da concorrência se verifica em Ramalho (2009, p. 59) e, particularmente no setor saúde, em Santos (2007).

Ao longo dos últimos anos, o número de beneficiários de planos de saúde cresceu significativamente, apresentando uma distinção marcante quanto ao tipo de contratação, que pode ser individual/familiar (doravante individual) ou coletiva, esta última com participação e crescimento mais significativos (ANS, 2007). A contratação coletiva em geral está relacionada ao mercado de trabalho pelo fato de ser o segmento no qual os contratantes são pessoas jurídicas, diferentemente do mercado individual, no qual os contratantes são pessoas físicas (LEAL e MATOS, 2007).

Os dois tipos de contratação também apresentam diferenciação relevante na regulação econômica dos planos de saúde. Existe uma dinâmica diferenciada em cada um desses mercados. No universo dos planos individuais, há uma tendência de que o poder de barganha dos beneficiários seja inferior ao dos planos coletivos, nos quais a negociação é feita por meio de uma pessoa jurídica contratante. Assim é que se justifica uma regulação menos intensa por parte do poder público para os planos coletivos, que possuem, por exemplo, reajustes de preços livres. Para os planos individuais, por sua vez, a ANS define um percentual máximo de reajuste anual com base na evolução dos reajustes do mercado coletivo. Essa diferenciação da amplitude e intensidade de regulação, bem como da evolução de beneficiários, suscita a importância da avaliação comparativa entre os tipos de contratação.

Dentre as principais discussões na área de saúde suplementar, e na esteira da temática dos reajustes, destaca-se a importância da avaliação dos custos em saúde. A relevância da evolução dos custos em saúde é um fenômeno mundial, num contexto de tendência de participação crescente no PIB das nações. Além disso, ressalta-se que no Brasil há evidências do peso crescente da saúde no orçamento das famílias e da elevada participação de seus produtos na formação das taxas dos índices de preço ao consumidor (BRAZ, 2006, p. 50).

De acordo com Porter (1980), três estratégias genéricas podem ser usadas individualmente ou em conjunto para criar uma posição sustentável a longo prazo: diferenciação, custo ou foco. A vantagem competitiva em saúde pode ser obtida por meio de estratégias de diferenciação ligadas a muitos fatores geradores de custos (estrutura de hotelaria e operacional, cuidado médico e cultura da organização, entre outros), que precisam ser administrados (PORTER e TEISBERG, 2007; KLOCK, 2009; NEVES, 2009). Segundo Lima e Lima (1998), alguns dos principais problemas das organizações de saúde são a ineficiência e os custos crescentes. Assim, torna-se essencial o aprimoramento da gestão hospitalar, para a administração de custos e eficiência na prestação de serviços de saúde, envolvendo aspectos como sistemas de informação, otimização das aquisições de insumos e controles de custos e de qualidade (MEDICI e MARQUES, 1996).

Nesse contexto, a motivação deste estudo, que é a apuração de informações acuradas sobre a evolução dos valores praticados no mercado de saúde suplementar, é uma importante contribuição para possibilitar a identificação de tendências e dos fatores mais relevantes (ZUCHI e outros, 2000), com vistas ao aprimoramento da gestão hospitalar, ao posicionamento estratégico das empresas (PORTER, 1980) ou à definição das políticas públicas do setor (MALIK, 1995).

Para a análise da evolução dos custos assistenciais em saúde, uma relevante diferenciação é a separação em seus dois componentes, a variação dos custos médios e a variação das quantidades. A variação dos custos médios (custos unitários) representa o aumento dos preços dos insumos (proxy da inflação) e o aumento da incorporação tecnológica cumulativa do setor. A variação das quantidades (frequência de utilização), por sua vez, pode ser associada aos argumentos da ampliação do cuidado com a saúde e do envelhecimento da população.

Nesse contexto, o objetivo do presente estudo é avaliar a evolução dos custos assistenciais dos planos de saúde, separadamente por seus dois componentes, custo médio e quantidade, considerando a distinção marcante entre os tipos de contratação. Para essa finalidade, foram utilizadas informações, de 2002 até 2006, do Sistema de Informações de Produtos (SIP) da ANS, divulgadas na página eletrônica dessa agência, que apresentam desagregação segundo o tipo de contrato e segundo categorias de serviços médicos. Este estudo estima, de forma pioneira para essa base de dados, os índices de variação com base na metodologia 
de números índices e na definição atuarial que indica que o custo por seguro (valor) pode ser separado na frequência de uso dos beneficiários (quantidade) e no custo dos serviços (preço). O trabalho está divido em três seções, além desta. A primeira apresenta a regulamentação do setor de saúde suplementar no que se refere às distinções das categorias de contratação, bem como alguns dados da evolução recente do setor. A segunda apresenta aspectos metodológicos fundamentais para a presente análise, como a teoria de números índices (valor, preço e quantidade) e a aplicação dessa metodologia nos custos em saúde suplementar. A terceira apresenta as evidências empíricas da evolução dos custos, quais sejam, a evolução dos custos médios (índice de valor) numa primeira etapa e a evolução dos componentes frequência de utilização (índice de quantidade) e custo médio por evento (índice de preço). Finalmente, uma seção de considerações finais aponta os principais itens analisados.

\section{PREÇOS E CUSTOS NO MERCADO DE PLANOS DE SAÚDE}

A saúde suplementar tem como marco regulatório a Lei $\mathrm{n}^{\circ} 9.656 / 1998$, combinada às medidas provisórias que a alteraram (atualmente em vigor a MP n ${ }^{\circ} 2.177-44 / 2001$ ). Do ponto de vista da regulação do produto, uma distinção marcante desse setor é o tipo de contratação, que pode ser individual ou coletivo. O contrato individual é aquele oferecido para a livre adesão do consumidor pessoa física. Conforme detalhado em ANS (2005), o contrato coletivo é aquele firmado por uma pessoa jurídica (empresa, associação, fundação ou sindicato), do qual é beneficiária uma pessoa na condição de empregado, associado ou sindicalizado, e apresenta duas modalidades:

i) o contrato coletivo por adesão se refere a planos em que a adesão dos beneficiários é opcional e espontânea;

ii) o plano coletivo empresarial, por sua vez, é contratado por uma pessoa jurídica para atender a uma massa populacional específica a ela vinculada, cuja adesão é automática e obrigatória, por meio de duas modalidades: ii.a) sem patrocínio, quando pago integralmente pelo beneficiário diretamente à operadora; ou

ii.b) com patrocínio, quando pago integral ou parcialmente pela pessoa jurídica à operadora, inclusive os casos de autogestão nos quais o beneficiário paga parte da mensalidade.
Conforme apresentadas em Leal e Matos (2008a, p. 8290), as principais características que diferenciam os planos individuais dos coletivos são a diversidade e a intensidade do arcabouço regulamentar, esta segunda mais estrita nos planos de contratação individual do que nos planos coletivos. A cobertura técnico-assistencial a ser oferecida pelas operadoras é invariante com a modalidade de contratação. Por outro lado, a regulação da utilização e a regra de reajuste de preços são assuntos que espelham diferencial entre os tipos de contratação. Sobre a regra de reajuste, destaca-se que a ANS estabelece o percentual de reajuste máximo a ser aplicado no mercado individual com base na evolução dos reajustes do mercado coletivo, que possui reajustes livres.

A título de contextualização, a Figura 1 mostra a predominância e o crescimento dos planos coletivos, bem como a melhoria das bases de dados da ANS, analisada com mais profundidade em Leal e Matos (2008b).

A Figura 2 ilustra a evolução, nos EUA, dos prêmios de seguro-saúde, em níveis superiores à inflação geral. No Brasil, diversos estudos empíricos comparam os índices de reajuste da ANS com índices de preços, como Saintive e Chacur (2006), Ocké-Reis e Cardoso (2006), Teixeira (2006) e Idec (2006). Entretanto, cabe ressaltar a observação metodológica de Leal e Matos (2008b) de que os índices de reajuste da ANS são índices de valor, e não índices de preço stricto sensu. Os autores especificam que esse índice da ANS contempla: i) efeitos de variações de aumento de preços; ii) frequência de utilização; iii) introdução de novas tecnologias. Paralelamente, as variações decorrentes da mudança de faixa etária dos beneficiários seguem dinâmica própria. Desse modo, as variações das mensalidades dos planos de saúde não são de natureza de preços, posto que consideram variações de preços e de quantidades. Neste estudo, conforme ilustrado na Figura 3, Leal e Matos (2008b) mostram que o modelo de regulação de reajustes da ANS tem conferido em geral reajustes superiores aos principais índices de preços e relativamente próximos da variação do PIB nominal, a principal referência de índice de valor da economia.

\section{DA METODOLOGIA DE MEDIÇÃO DA VARIAÇÃO DOS CUSTOS SEGUNDO SEUS COMPONENTES PREÇO E QUANTIDADE}

Inicialmente, cabe registrar a metodologia utilizada pelo IBGE (2007) para a mensuração da evolução dos custos 
na construção civil, no Sinapi, em que é utilizado um índice de valor (denominado mais usualmente índice de custo), conforme ilustrado no Quadro 1.
Observa-se que o índice de valor compara a multiplicação dos preços (p) e quantidades (q) no período $t$ com seu respectivo valor no período 0 . A diferença com os índices

Figura 1 - Beneficiários de planos médicos segundo o tipo de contratação

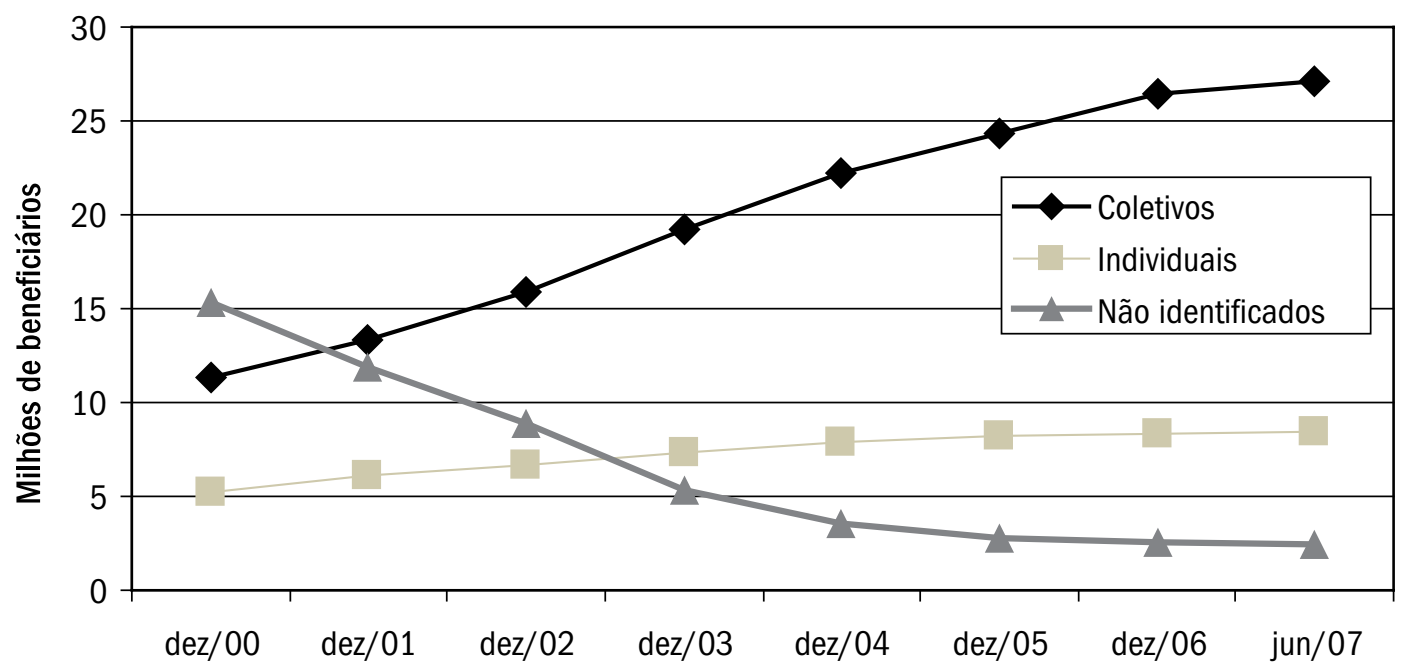

Fonte: Elaboração própria, com base em ANS (2007).

Figura 2 - Evolução dos prêmios de seguro-saúde nos EUA

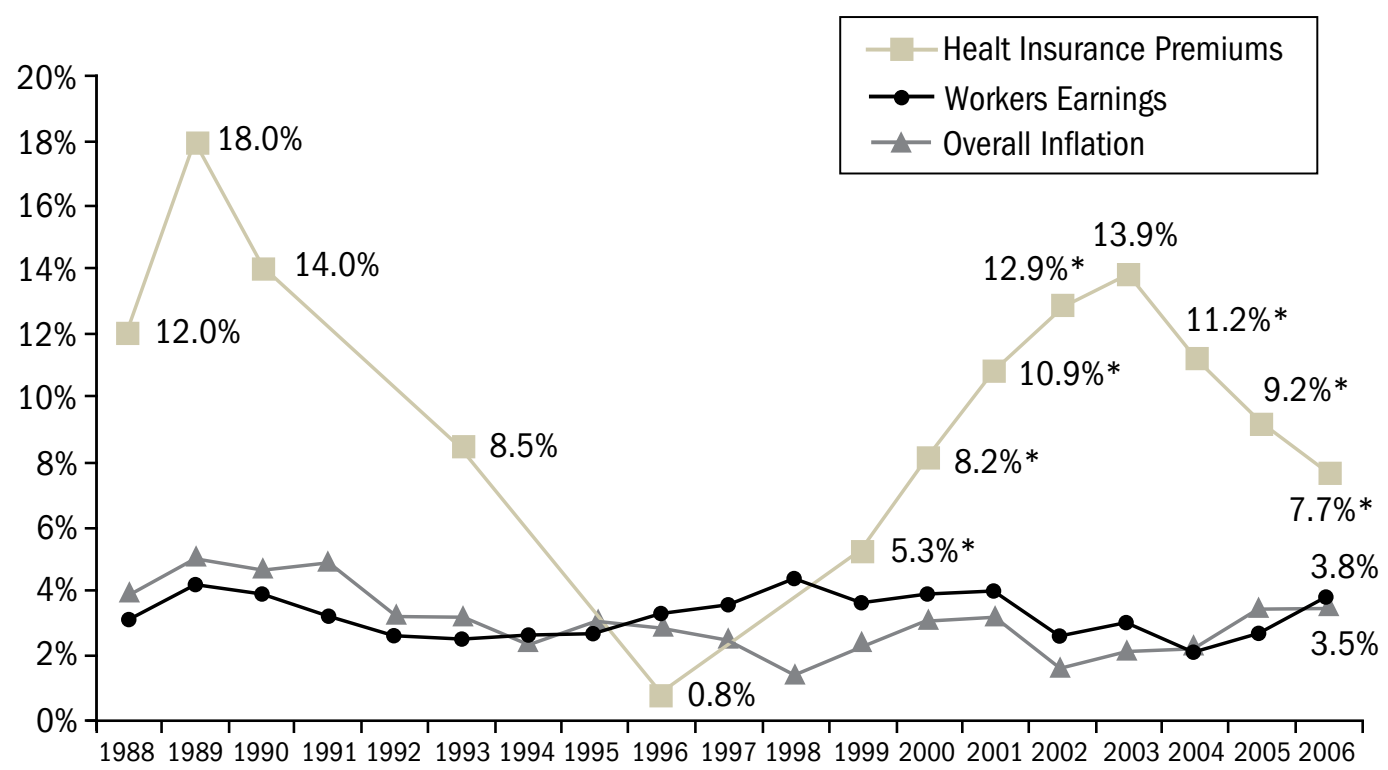

Fonte: Kaiser/HRET (2006, p.3).

Nota: Prêmios de seguro-saúde (health insurance premiums); ganhos salariais dos trabalhadores (worker earnings); inflação geral (overall inflation). 
de preços consiste basicamente no fato de que esses representam a evolução dos preços considerando a quantidade como base fixa, seja a quantidade do período 0 (Laspeyres) ou a quantidade do período $t$ (Paasche). A diferença com os índices de quantidade é análoga, só que nesse caso os preços é que são considerados como base fixa.

Essa publicação do IBGE (2007) aborda ainda o índice de Fischer (média geométrica dos índices de Laspeyres e Paasche), bem como a forma de cálculo do índice de valor por meio da multiplicação de índices de quantidade e de índices de preço, conforme exposto: "A multiplicação do índice de preço de Laspeyres pelo índice de quantidade de Paasche ( $1^{a}$ hipótese) ou do índice de preço de Paasche pelo índice de quantidade de Laspeyres ( $2^{\mathrm{a}}$ hipótese) possibilita obter o índice de valor".

É possível aplicar essa teoria de que o índice de valor possui como componentes índices de preço e de quantidade ao setor de saúde suplementar. Como exemplo, cabe registrar que o índice de reajuste defi- nido pela ANS para aplicação no mercado individual consiste num índice de valor (preço multiplicado por quantidade, portanto).

No caso das despesas com assistência médica, os dois componentes são a evolução da quantidade utilizada e a evolução dos custos médios com os procedimentos médicos. No contexto da saúde suplementar, são utilizados principalmente dois indicadores que refletem esses componentes (ANS, 2007, p. 31). O primeiro é o custo médio dos eventos ( $\mathrm{CmEv})$, que é a relação entre a despesa nos eventos (DeEv) e o número de eventos (Ev), e reflete, em tese, a evolução dos preços médios dos insumos (procedimentos médicos), inclusive a inflação médica e a variação tecnológica, isolando esse montante do aumento do impacto da quantidade utilizada. O segundo é a frequência média de utilização (FrUt), que é a relação entre o número de eventos (Ev) e o número de expostos (Ex), e reflete, em alguma medida, o aumento do grau de utilização dos usuários que têm direito à utilização dos

Figura 3 - Evolução dos reajustes autorizados pela ANS* e do PIB nominal

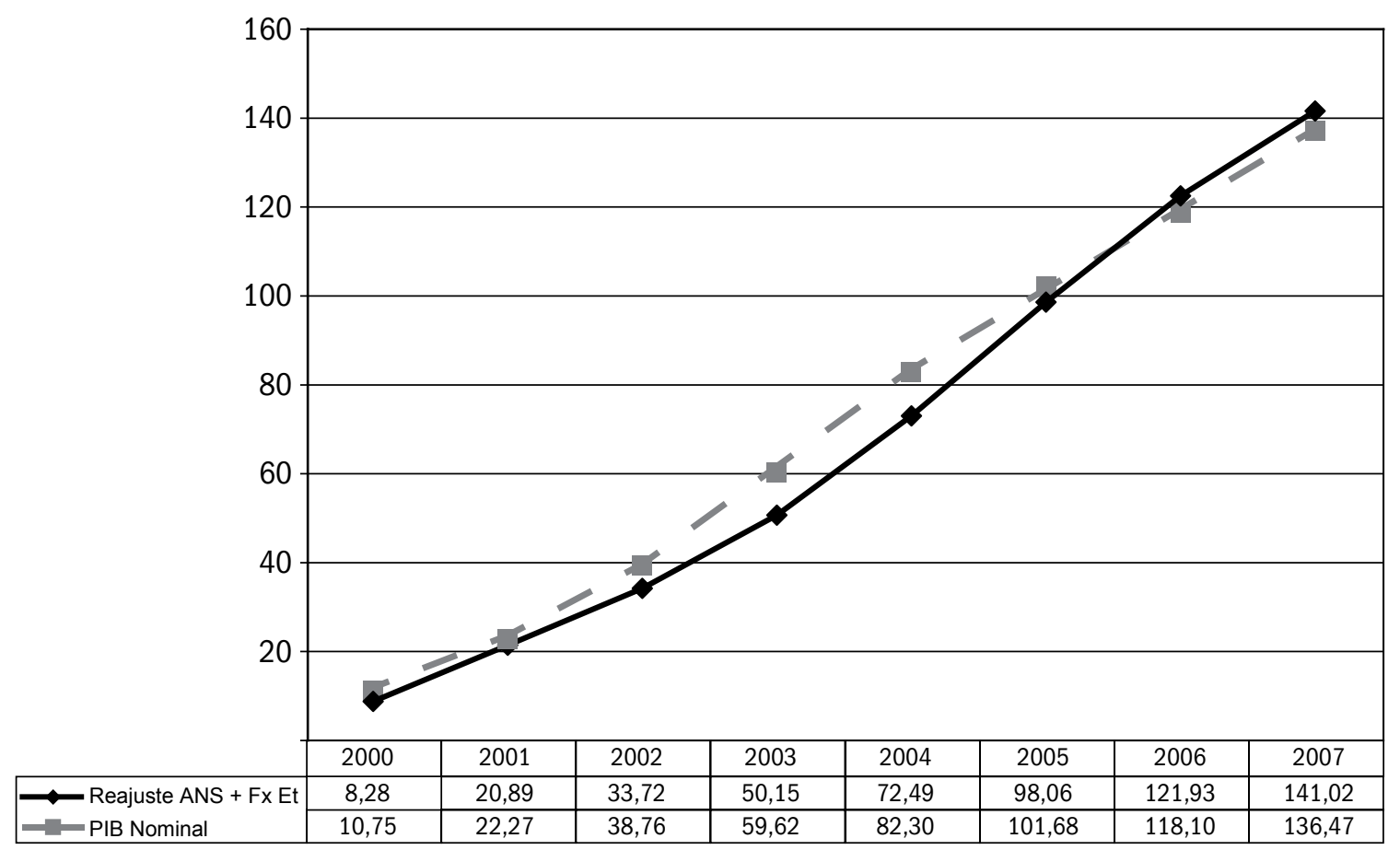

Fonte: Leal e Matos (2008b, p.10).

Nota: Elaboração própria, com dados do Bacen e TabNet-ANS. Previsão de crescimento real do PIB de 5,2\% em 2007 (estimativa do Banco Central em fevereiro de 2008). Índice de reajuste autorizado pela ANS calculado utilizando o período de aplicação. Estimativa própria do impacto de variação de faixa etária. 
serviços médicos. Em resumo, os dois indicadores são calculados desta forma:

$$
\mathrm{CmEv}=\frac{\mathrm{DeEv}}{\mathrm{Ev}}
$$

$$
\text { FrUt }=\frac{\mathrm{Ev}}{\mathrm{Ex}}
$$

Esses dois indicadores são divulgados pela ANS (2007) com base no Sistema de Informações de Produtos (SIP), segundo o tipo de contratação (individual, coletivo com patrocínio e coletivo sem patrocínio) e segundo o tipo de evento (para os planos médicos, são as consultas médicas, as internações, os exames complementares, as terapias e os outros atendimentos ambulatoriais). Sobre a implementação da regulação no setor, e especificamente sobre o SIP, mais detalhes em Santos (2007).

Há também um terceiro indicador, o custo médio por exposto (CmEx), que é a relação entre a despesa nos eventos (DeEv) e o número de expostos (Ex), ou seja, visa representar a despesa média dos usuários que têm direito à utilização dos serviços médicos. Dentre os indicadores analisados, este é o mais próximo da despesa por beneficiário, e, nesse contexto, pode ser comparado com a evolução da receita média por beneficiário, ou preço, dos planos de saúde. Em termos algébricos, é possível calcular o custo médio por exposto por meio da multiplicação da frequência de utilização pelo custo médio por evento, conforme demonstrado a seguir:

$$
\frac{\mathrm{DeEv}}{\mathrm{Ex}}=\frac{\mathrm{Ev}}{\mathrm{Ex}} \mathrm{x} \frac{\mathrm{DeEv}}{\mathrm{Ev}}
$$

$$
\mathrm{CmEx}=\text { FrUt } \mathrm{x} \text { CmEv }
$$

Antes de avaliar a evolução dos custos, cabe registrar que a estrutura das despesas dos planos de saúde pode ser separada, de forma simplificada, entre as despesas assistenciais, que são relacionadas diretamente à utilização dos serviços de saúde, e as despesas não assistenciais, como as despesas de comercialização, administrativas e outras. O objeto da presente análise são as despesas assistenciais dos planos de saúde médicos. Os dados divulgados pela ANS permitem sua investigação por meio das informações contábeis ou das informações do Sistema de Informações de Produtos (SIP), que são específicas de custos e utilização dos planos de saúde. Destaca-se que, das informações divulgadas, somente as informações do SIP, diferentemente das informações contábeis, permitem a segmentação por produto segundo o tipo de contratação.

\section{RESULTADOS DA EVOLUÇÃO DOS CUSTOS ASSISTENCIAIS}

Tendo em vista o exposto na seção de metodologia, a análise empírica é realizada em duas etapas. Na primeira, são apresentadas as informações da evolução do custo médio por exposto (calculado com dados do SIP), comparativamente à despesa assistencial por beneficiário (calculada com dados contábeis). Na segunda, integralmente com

\begin{tabular}{|c|c|c|}
\hline ÍNDICE & LASPEYRES & PAASCHE \\
\hline Preços & $\frac{\sum_{i} p_{i, t} \cdot q_{i, 0}}{\sum_{i} p_{i, 0} \cdot q_{i, 0}}$ & $\frac{\sum_{i} p_{i, t} \cdot q_{i, t}}{\sum_{i} p_{i, 0} \cdot q_{i, t}}$ \\
\hline Quantidade & $\frac{\sum_{i} p_{i, 0} \cdot q_{i, t}}{\sum_{i} p_{i, 0} \cdot q_{i, 0}}$ & $\frac{\sum_{i} p_{i, t} \cdot q_{i, t}}{\sum_{i} p_{i, t} \cdot q_{i, 0}}$ \\
\hline Valor & & \\
\hline
\end{tabular}
dados do SIP, é avaliada a evolução dos componentes do custo médio por exposto, que são a frequência de utilização (índice de quantidade) e o custo médio por evento (índice de preço).

Quadro 1 - Especificação dos índices de preços, quantidades e valor

Fonte: Elaboração própria, com base em IBGE (2007). 


\section{Evolução dos custos assistenciais}

Inicialmente, estimou-se com base nos dados contábeis a evolução da despesa assistencial por beneficiário, conforme apresentada na Tabela 1 .

Em segundo lugar, com base nos dados do SIP, calculouse o custo médio por exposto, para cada um dos tipos de procedimentos. Em seguida, estimou-se o custo médio por exposto em todos os procedimentos, por meio da simples soma dos valores para cada um dos cinco tipos de procedimentos. A título de ilustração, tais estimativas são apresentadas na Tabela 2.

Ao analisar os dados em nível segundo o tipo de contratação, observa-se que, em todos os períodos, a despesa por exposto dos planos coletivos com patrocínio é a que apresenta menores níveis, seguida pelos planos individuais, enquanto os planos coletivos sem patrocínio apresentam os maiores níveis de despesa por exposto. Observando os dados segundo o tipo de procedimento, percebe-se que os menores níveis de despesa por exposto para os planos coletivos com patrocínio são verdadeiros para todos os cinco tipos de procedimentos.

Já na análise da variação acumulada, observa-se que, em termos de despesa por exposto geral, foi bastante próxima para os três tipos de contratação. Ao avaliar as informações do segundo tipo de procedimento, destaca-se que a maior diferenciação foi identificada nos outros atendimentos ambulatoriais, que representam uma parcela reduzida do total da despesa. Para os três tipos de contratação, foi mais significativa a variação acumulada das terapias. A menor variação acumulada, por sua vez, foi identificada em exames complementares, para os planos sem patrocínio, e em outros atendimentos ambulatoriais, para os planos individuais e para os coletivos com patrocínio.

Ao comparar com a evolução das despesas assistenciais das informações contábeis das operadoras, apresentadas anteriormente, observa-se que os níveis de despesas assistenciais por beneficiário (ao ano) não apresentam distinção marcante com os níveis de custo geral por exposto para os planos individuais e coletivos com patrocínio, o que é positivo para a validade desse conjunto de dados. Quanto à variação acumulada, de 2002 a 2006, foi superior para a despesa assistencial contábil por beneficiário (36,6\%), em relação ao custo geral médio por exposto para qualquer um dos três tipos de contratação.

\section{Evolução dos custos assistenciais segundo os componentes preço e quantidade}

Com o objetivo de aprofundar a presente análise utilizando as informações de frequência de utilização e custo médio por evento, foram calculados os índices de variação para os diversos tipos de procedimentos médicos. Além disso, estimou-se o índice de variação agregado, considerando para a ponderação o peso do custo médio por exposto daquele procedimento em relação à soma do custo médio por exposto, que representa o peso de cada procedimento nos gastos do usuário médio. Desse modo,

Tabela 1 - Evolução da despesa assistencial das operadoras médico-hospitalares

\begin{tabular}{|c|c|c|c|c|c|}
\hline \multirow{2}{*}{\multicolumn{2}{|c|}{ INFORMAÇÃO }} & \multirow[t]{2}{*}{$\begin{array}{l}\text { DESPESA ASSISTENCIAL } \\
\text { (1) - R\$ }\end{array}$} & \multirow[t]{2}{*}{ BENEFICIÁRIOS (2) } & \multicolumn{2}{|c|}{$\begin{array}{l}\text { DESPESA ASSISTENCIAL POR } \\
\text { BENEFICIÁRIO - R\$ }\end{array}$} \\
\hline & & & & ANUAL & MENSAL \\
\hline \multirow{6}{*}{ Período } & 2001 & 17.151.926.014 & 31.287 .966 & 548 & 46 \\
\hline & 2002 & 19.949 .211 .972 & 31.368 .085 & 636 & 53 \\
\hline & 2003 & 22.680 .579 .881 & 31.893 .487 & 711 & 59 \\
\hline & 2004 & 25.665 .291 .661 & 33.583 .357 & 764 & 64 \\
\hline & 2005 & 28.668 .306 .523 & 35.335 .258 & 811 & 68 \\
\hline & 2006 & 32.461 .699 .143 & 37.366 .085 & 869 & 72 \\
\hline \multirow{4}{*}{ Indicador } & Valor médio & 24.429 .502 .532 & 33.472 .373 & 723 & 60 \\
\hline & Desvio padrão & 5.658 .721 .947 & 2.463 .177 & 117 & 10 \\
\hline & Coeficiente de variação & $23,2 \%$ & $7,4 \%$ & $16,2 \%$ & $16,2 \%$ \\
\hline & Variação Acumulada & $89,26 \%$ & $19,43 \%$ & $58,47 \%$ & $58,47 \%$ \\
\hline
\end{tabular}

Fonte: Elaboração própria, com dados da ANS (2007), originados do SIB (06/2007) e do Diops/FIP (04/06/2007).

Notas: (1) Não são incluídas as Autogestões patrocinadas.

(2) Informações correspondentes aos meses de dezembro. 
foram obtidos os resultados nas Tabelas 3 e 4.

As informações da Tabela 3 indicam que a frequência de utilização apresentou crescimento mais significativo para os planos coletivos com patrocínio, especialmente em 2005 e 2006, em relação aos outros dois tipos de planos. Ao avaliar as informações para os tipos de procedimento, infere-se que os três tipos de contratação obtiveram variação relativamente próxima, resultando nos mesmos destaques, que são as terapias, como maior variação, e outros atendimentos ambulatoriais, como diminuição mais intensa.

Conforme se pode ver na Tabela 4, o custo geral médio por evento apresentou maior variação acumulada, com pe-

Tabela 2 - Custo médio por exposto, segundo o tipo de evento de assistência médica e o tipo de contratação do plano

\begin{tabular}{|c|c|c|c|c|c|c|c|c|c|}
\hline TIPO DE EVENTO & 2002 & 2003 & 2004 & 2005 & 2006 & $\begin{array}{l}\text { VARIAÇÃO } \\
\text { ACUMULADA }\end{array}$ & MÉDIA & $\begin{array}{l}\text { DESVIO } \\
\text { PADRÃOO }\end{array}$ & $\begin{array}{c}\text { COEF. DE } \\
\text { VARIAÇÃ̃O }\end{array}$ \\
\hline \multicolumn{10}{|l|}{ Consultas médicas } \\
\hline Individual e familiar & 133 & 134 & 146 & 162 & 179 & $34,9 \%$ & 151 & 20 & $13,0 \%$ \\
\hline Coletivo com patrocínio & 102 & 104 & 112 & 128 & 142 & $39,5 \%$ & 118 & 17 & $14,5 \%$ \\
\hline Coletivo sem patrocínio & 129 & 137 & 152 & 163 & 174 & $34,8 \%$ & 151 & 18 & $12,2 \%$ \\
\hline \multicolumn{10}{|l|}{ Internações } \\
\hline Individual ou familiar & 271 & 294 & 330 & 326 & 407 & $50,5 \%$ & 326 & 52 & $15,9 \%$ \\
\hline Coletivo com patrocínio & 203 & 235 & 245 & 256 & 300 & $47,4 \%$ & 248 & 35 & $14,1 \%$ \\
\hline Coletivo sem patrocínio & 301 & 334 & 377 & 390 & 435 & $44,6 \%$ & 367 & 52 & $14,1 \%$ \\
\hline \multicolumn{10}{|l|}{ Exames complementares } \\
\hline Individual ou familiar & 182 & 184 & 201 & 223 & 234 & $28,5 \%$ & 205 & 23 & $11,3 \%$ \\
\hline Coletivo com patrocínio & 133 & 140 & 150 & 165 & 181 & $36,1 \%$ & 154 & 19 & $12,6 \%$ \\
\hline Coletivo sem patrocínio & 203 & 223 & 231 & 249 & 261 & $28,6 \%$ & 234 & 23 & $9,7 \%$ \\
\hline \multicolumn{10}{|l|}{ Terapias } \\
\hline Individual ou familiar & 39 & 51 & 55 & 74 & 72 & $82,7 \%$ & 58 & 15 & $25,4 \%$ \\
\hline Coletivo. com patrocínio & 26 & 32 & 38 & 46 & 50 & $94,2 \%$ & 38 & 10 & $25,8 \%$ \\
\hline Coletivo sem patrocínio & 49 & 59 & 62 & 84 & 83 & $67,4 \%$ & 67 & 15 & $22,7 \%$ \\
\hline \multicolumn{10}{|l|}{$\begin{array}{l}\text { Outros atendimentos } \\
\text { ambulatoriais }\end{array}$} \\
\hline Individual ou familiar & 55 & 59 & 68 & 57 & 70 & $27,2 \%$ & 62 & 7 & $10,8 \%$ \\
\hline Coletivo com patrocínio & 42 & 44 & 48 & 48 & 51 & $21,8 \%$ & 47 & 4 & $7,9 \%$ \\
\hline Coletivo sem patrocínio & 53 & 53 & 61 & 66 & 75 & $41,4 \%$ & 61 & 9 & $15,0 \%$ \\
\hline \multicolumn{10}{|l|}{ Geral } \\
\hline Individual ou familiar & 679 & 723 & 799 & 843 & 961 & $41,5 \%$ & 801 & 110 & $13,7 \%$ \\
\hline Coletivo com patrocínio & 506 & 556 & 593 & 643 & 724 & $43,1 \%$ & 604 & 84 & $13,8 \%$ \\
\hline Coletivo sem patrocínio & 735 & 806 & 884 & 952 & 1.027 & $39,8 \%$ & 881 & 116 & $13,1 \%$ \\
\hline
\end{tabular}

Fonte: Elaboração própria, com dados de ANS (2007), baseados no Sistema de Informações de Produtos (SIP), ANS/MS - 04/2007. 
quena magnitude de diferença, para os planos individuais em relação aos outros dois tipos de planos. Ao avaliar as informações para os tipos de procedimento, infere-se que os três tipos de contratação obtiveram variação relativamente próxima. Destacam-se, com a maior variação acumula$\mathrm{da}$, as internações, para os planos individuais e coletivos com patrocínio, enquanto para os planos sem patrocínio a maior variação foi o custo médio por evento do item outros atendimentos ambulatoriais. Com menores variações acumuladas de custo médio por evento, ressaltam-se o tipo de procedimento terapias, para todos os tipos de contratação, e o procedimento exames complementares, especialmente para os planos coletivos sem patrocínio.

Por fim, com o objetivo de facilitar a avaliação compara-

Tabela 3 - Variação da frequência média de utilização, segundo o tipo de evento de assistência médica e o tipo de contratação do plano

\begin{tabular}{|c|c|c|c|c|c|}
\hline TIPO DE EVENTO & 2003 & 2004 & 2005 & 2006 & $\begin{array}{l}\text { VARIAÇÃO } \\
\text { ACUMULADA }\end{array}$ \\
\hline \multicolumn{6}{|l|}{ Consultas médicas } \\
\hline Individual e familiar & $-4,2 \%$ & $1,2 \%$ & $0,9 \%$ & $2,7 \%$ & $0,4 \%$ \\
\hline Coletivo com patrocínio & $-2,3 \%$ & $0,2 \%$ & $3,5 \%$ & $4,0 \%$ & $5,3 \%$ \\
\hline Coletivo sem patrocínio & $0,3 \%$ & $3,6 \%$ & $-1,5 \%$ & $0,2 \%$ & $2,6 \%$ \\
\hline \multicolumn{6}{|l|}{ Internações } \\
\hline Individual ou familiar & $-8,7 \%$ & $-1,6 \%$ & $-8,0 \%$ & $7,0 \%$ & $-11,6 \%$ \\
\hline Coletivo com patrocínio & $-0,9 \%$ & $-8,3 \%$ & $-5,0 \%$ & $4,0 \%$ & $-10,2 \%$ \\
\hline Coletivo sem patrocínio & $-2,7 \%$ & $-5,2 \%$ & $-10,4 \%$ & $4,2 \%$ & $-14,0 \%$ \\
\hline \multicolumn{6}{|l|}{ Exames complementares } \\
\hline Individual ou familiar & $-4,8 \%$ & $3,8 \%$ & $19,8 \%$ & $2,7 \%$ & $21,6 \%$ \\
\hline Coletivo com patrocínio & $2,5 \%$ & $5,2 \%$ & $19,3 \%$ & $3,4 \%$ & $33,1 \%$ \\
\hline Coletivo sem patrocínio & $2,8 \%$ & $0,8 \%$ & $20,4 \%$ & $4,2 \%$ & $29,9 \%$ \\
\hline \multicolumn{6}{|l|}{ Terapias } \\
\hline Individual ou familiar & $14,3 \%$ & $11,2 \%$ & $24,3 \%$ & $13,3 \%$ & $79,0 \%$ \\
\hline Coletivo com patrocínio & $20,1 \%$ & $17,9 \%$ & $21,3 \%$ & $14,4 \%$ & $96,6 \%$ \\
\hline Coletivo sem patrocínio & $6,1 \%$ & $12,6 \%$ & $13,0 \%$ & $22,6 \%$ & $65,6 \%$ \\
\hline \multicolumn{6}{|c|}{ Outros atendimentos ambulatoriais } \\
\hline Individual ou familiar & $-0,06$ & 0,00 & $-0,25$ & 0,09 & $-23,3 \%$ \\
\hline Coletivo com patrocínio & $-0,05$ & $-0,03$ & $-0,16$ & 0,05 & $-18,8 \%$ \\
\hline Coletivo sem patrocínio & $-0,13$ & $-0,03$ & $-0,14$ & 0,07 & $-22,0 \%$ \\
\hline \multicolumn{6}{|l|}{ Geral } \\
\hline Individual ou familiar & $-5,0 \%$ & $1,2 \%$ & $2,8 \%$ & $5,7 \%$ & $4,5 \%$ \\
\hline Coletivo com patrocínio & $0,6 \%$ & $-1,2 \%$ & $4,0 \%$ & $4,6 \%$ & $8,1 \%$ \\
\hline Coletivo sem patrocínio & $-0,7 \%$ & $-0,7 \%$ & $1,0 \%$ & $5,2 \%$ & $4,7 \%$ \\
\hline
\end{tabular}

Fonte: Elaboração própria, com dados de ANS (2007), baseados no Sistema de Informações de Produtos (SIP), ANS/MS - 04/2007. 
tiva, os resultados expostos foram sintetizados na Tabela 5.

Assim, observa-se de modo mais evidente, para as três categorias de contratação, que, dentre os dois componentes do custo médio por exposto (índice de valor), houve aumento mais intenso do custo médio dos eventos (índice de preço) em relação à frequência de utilização (índice de quantidade).

\section{CONSIDERAÇÕES FINAIS}

Tendo em vista a relevância da discussão dos custos crescentes em saúde, é de suma importância aprofundar as investigações sobre os custos assistenciais em saúde suplementar no Brasil. Nesse contexto, paralelamente à análise do comportamento dos grupos de procedimentos

Tabela 4 - Variação do custo médio dos eventos médico-hospitalares, segundo o tipo de evento e de contratação do plano

\begin{tabular}{|c|c|c|c|c|c|}
\hline TIPO DE EVENTO & 2003 & 2004 & 2005 & 2006 & $\begin{array}{c}\text { VARIAÇÃO } \\
\text { ACUMULADA }\end{array}$ \\
\hline \multicolumn{6}{|l|}{ Consultas médicas } \\
\hline Individual e familiar & $5,9 \%$ & $7,6 \%$ & $10,0 \%$ & $7,1 \%$ & $34,3 \%$ \\
\hline Coletivos com patrocínio & $4,8 \%$ & $7,3 \%$ & $10,5 \%$ & $6,5 \%$ & $32,5 \%$ \\
\hline Coletivo sem patrocínio & $5,8 \%$ & $7,1 \%$ & $8,9 \%$ & $6,6 \%$ & $31,4 \%$ \\
\hline \multicolumn{6}{|l|}{ Internações } \\
\hline Individual ou familiar & $19,1 \%$ & $13,8 \%$ & $7,5 \%$ & $16,8 \%$ & $70,1 \%$ \\
\hline Coletivo com patrocínio & $16,8 \%$ & $13,4 \%$ & $10,0 \%$ & $12,7 \%$ & $64,3 \%$ \\
\hline Coletivo sem patrocínio & $14,2 \%$ & $19,2 \%$ & $15,4 \%$ & $7,0 \%$ & $68,1 \%$ \\
\hline \multicolumn{6}{|l|}{ Exames complementares } \\
\hline Individual ou familiar & $6,3 \%$ & $4,9 \%$ & $-7,1 \%$ & $1,9 \%$ & $5,7 \%$ \\
\hline Coletivo com patrocínio & $2,7 \%$ & $1,8 \%$ & $-7,7 \%$ & $6,1 \%$ & $2,3 \%$ \\
\hline Coletivo sem patrocínio & $7,1 \%$ & $2,9 \%$ & $-10,5 \%$ & $0,4 \%$ & $-1,0 \%$ \\
\hline \multicolumn{6}{|l|}{ Terapias } \\
\hline Individual ou familiar & $3,3 \%$ & $3,2 \%$ & $2,7 \%$ & $2,8 \%$ & $2,1 \%$ \\
\hline Coletivo com patrocínio & $3,2 \%$ & $2,9 \%$ & $2,6 \%$ & $2,3 \%$ & $-1,2 \%$ \\
\hline Coletivo sem patrocínio & $3,2 \%$ & $3,1 \%$ & $2,5 \%$ & $2,6 \%$ & $1,1 \%$ \\
\hline \multicolumn{6}{|c|}{ Outros atendimentos ambulatoriais } \\
\hline Individual ou familiar & $15,4 \%$ & $14,4 \%$ & $11,6 \%$ & $12,7 \%$ & $66,0 \%$ \\
\hline Coletivo com patrocínio & $10,6 \%$ & $13,3 \%$ & $19,0 \%$ & $0,7 \%$ & $50,0 \%$ \\
\hline Coletivo sem patrocínio & $16,3 \%$ & $17,9 \%$ & $25,9 \%$ & $5,1 \%$ & $81,4 \%$ \\
\hline \multicolumn{6}{|l|}{ Geral } \\
\hline Individual ou familiar & $12,0 \%$ & $9,8 \%$ & $4,0 \%$ & $10,0 \%$ & $40,6 \%$ \\
\hline Coletivo com patrocínio & $9,7 \%$ & $8,6 \%$ & $5,7 \%$ & $8,3 \%$ & $36,4 \%$ \\
\hline Coletivo sem patrocínio & $10,1 \%$ & $11,6 \%$ & $7,1 \%$ & $4,7 \%$ & $37,9 \%$ \\
\hline
\end{tabular}

Fonte: Elaboração própria, com dados de ANS (2007), baseados no Sistema de Informações de Produtos (SIP), ANS/MS - 04/2007. 
médicos, a aplicação da metodologia dos dois componentes dos índices de valor (índice de preço e índice de quantidade) se constitui num instrumento bastante útil para a averiguação dos principais determinantes da variação das despesas, seja no segmento privado ou no público.

O primeiro componente, o índice de preços (custo médio por evento), representa o aumento dos custos unitários e pode ser determinado principalmente pela inflação dos insumos e pela incorporação tecnológica. $\mathrm{O}$ índice de quantidade (frequência de utilização), por sua vez, representa o incremento de utilização pelos beneficiários, que pode ter relação com algumas categorias de fatores, tais quais: a) sociais, como a popularização dos serviços (especialmente pela inclusão das regiões mais pobres) e a generalização dos procedimentos (num contexto de ampliação dos cuidados para a saúde); b) demográficos, como o aumento da participação dos idosos - que tendem a uma utilização mais intensa - na população; e c) perfil epidemiológico.

A avaliação, em geral, dos três tipos de contratação (individual, coletivo com patrocínio e coletivo sem patrocínio), possibilitou observar que as variações de valor apresentaram trajetória crescente, corroborando a expectativa com base no comportamento internacional dos gastos em saúde. A análise segundo os dois componentes apontou que as variações dos índices de preço (custo médio por evento) foram superiores às dos índices de quantidade (frequência de utilização), em todo o período de 2002 a 2006. No que se refere à trajetória de evolução no tempo, o índice de preço apresentou alguma oscilação, enquanto o índice de quantidade revelou uma taxa de crescimento mais intensa.
No período analisado, infere-se que, embora o custo médio por exposto (índice de valor) apresente evolução próxima para os três tipos de contratação, há diferença quando são analisados separadamente seus dois componentes, a frequência de utilização (quantidade) e custo médio por evento (preço). Os planos coletivos com patrocínio apresentaram uma distinção em relação a outras categorias de contratação, pois obtiveram uma maior e mais destacada variação na frequência de utilização, ao mesmo tempo que obtiveram a menor variação, com pequena diferença de magnitude, no custo médio por evento.

Neste ponto, cabe registrar uma limitação metodológica da presente estimativa, pois com os dados analisados não foi possível decompor o índice de preços segundo os efeitos de variação de preços na cesta de procedimentos médicos vigentes de modo isolado dos efeitos da incorporação tecnológica. Um possível caminho para superar esse último desafio é a avaliação da compatibilidade dos dados da ANS com os dados de índices de preços oficiais, considerando a mencionada distinção entre os índices de valor e seus dois componentes. Além disso, a relação da evolução dos custos com o perfil etário dos beneficiários de planos de saúde não pôde ser explorada, uma vez que os dados da ANS não apresentam a desagregação das duas modalidades de planos coletivos (com ou sem patrocínio).

Por fim, como recomendação para pesquisas futuras, vale investigar os possíveis determinantes dessa maior relevância para a variação do índice de preços em relação à variação do índice de quantidades. Em específico, algumas hipóteses explicativas podem ser aprofundadas, especialmente se o crescimento mais intenso da trajetó-

Tabela 5 - Variação dos indicadores de custo médio por exposto e seus componentes: frequência de utilização e custo médio dos eventos médico-hospitalares

\begin{tabular}{|c|c|c|c|c|c|c|}
\hline \multirow[t]{2}{*}{ INDICADOR } & \multirow[t]{2}{*}{ TIPO DE CONTRATAÇÃO } & \multicolumn{5}{|c|}{ VARIAÇÃO \% } \\
\hline & & 2003 & 2004 & 2005 & 2006 & ACUMULADO \\
\hline \multirow{3}{*}{ Frequência de utilização } & Individual ou familiar & $-5,0 \%$ & $1,2 \%$ & $2,8 \%$ & $5,7 \%$ & $4,5 \%$ \\
\hline & Coletivo com patrocínio & $0,6 \%$ & $-1,2 \%$ & $4,0 \%$ & $4,6 \%$ & $8,1 \%$ \\
\hline & Coletivo sem patrocínio & $-0,7 \%$ & $-0,7 \%$ & $1,0 \%$ & $5,2 \%$ & $4,7 \%$ \\
\hline \multirow{3}{*}{$\begin{array}{l}\text { Custo médio dos } \\
\text { eventos médicos }\end{array}$} & Individual ou familiar & $12,0 \%$ & $9,8 \%$ & $4,0 \%$ & $10,0 \%$ & $40,6 \%$ \\
\hline & Coletivo com patrocínio & $9,7 \%$ & $8,6 \%$ & $5,7 \%$ & $8,3 \%$ & $36,4 \%$ \\
\hline & Coletivo sem patrocínio & $10,1 \%$ & $11,6 \%$ & $7,1 \%$ & $4,7 \%$ & $37,9 \%$ \\
\hline \multirow{3}{*}{ Custo médio por exposto } & Individual ou familiar & $6,5 \%$ & $10,5 \%$ & $5,5 \%$ & $14,0 \%$ & $41,5 \%$ \\
\hline & Coletivo com patrocínio & $9,8 \%$ & $6,8 \%$ & $8,4 \%$ & $12,5 \%$ & $43,1 \%$ \\
\hline & Coletivo sem patrocínio & $9,7 \%$ & $9,7 \%$ & $7,8 \%$ & $7,8 \%$ & $39,8 \%$ \\
\hline
\end{tabular}

Fonte: Elaboração própria, com dados de ANS (2007), baseados no Sistema de Informações de Produtos (SIP), ANS/MS - $04 / 2007$. 
ria da quantidade utilizada, no tempo, for um resultado esperado do aprofundamento do acesso aos serviços de saúde, num contexto de melhoria social com a generalização dos serviços e de progressão demográfica com o envelhecimento do perfil etário. Além disso, outra recomendação é investigar a importância do aumento relativo das doenças crônico-degenerativas, associadas de modo geral à utilização intensa e periódica dos serviços, num contexto de transição incompleta na direção do padrão epidemiológico dos países desenvolvidos, pois ainda persiste a relevância da morbidade-mortalidade por motivo das doenças infecciosas e de causas externas.

\section{REFERÊNCIAS}

AGÊNCIA NACIONAL DE SAÚDE SUPLEMENTAR. Reajuste de mensalidade: Conceitos básicos, reajuste por variação de custos, reajuste por mudança de faixa etária. 2. ed. rev. ampl, 2005.

AGÊNCIA NACIONAL DE SAÚDE SUPLEMENTAR. Carência, doenças e lesões preexistentes, urgência e emergência: prazos de carência, chequecaução, preenchimento da declaração de saúde. 3. ed. rev. ampl, 2005b.

AGÊNCIA NACIONAL DE SAÚDE SUPLEMENTAR. Caderno de informação da saúde suplementar: beneficiários, operadoras e planos. Setembro, 2007

BRAZ, A. A saúde no orçamento familiar. Conjuntura Econômica, FGV, v. 60, n. 4 , p. 50, 2006.

BRASIL. Lei n. 9.656, de 3 de junho de 1998. Atualizada até a medida provisória n ${ }^{\circ} 2.177-44$, de 24 de agosto de 2001. Dispõe sobre os planos e seguros provados de assistência à saúde.

IBGE. Sistema Nacional de Pesquisa de Custos e Índices da Construção Civil - O índice: visão teórica. Disponível em http://www.ibge.gov.br/home/ estatistica/indicadores/precos/sinapi/indice.shtm. Acesso em 09.11.2007.

INSTITUTO BRASILEIRO DE DEFESA DO CONSUMIDOR. Estudo sobre os reajustes anuais de planos de saúde e a atuação da ANS na regulação dessa questão. São Paulo, 2006.

KAISER FAMILY FOUNDADTION. HEALTH RESEARCH \& EDUCATIONAL TRUST. Survey of Employer Health Benefits 2006. Annual Survey. 2006. Disponível em: http://www.kff.org/insurance/7527/ upload/7561.pdf. Acesso em 01.07.2009.

KLOCK, J. Obtendo vantagem competitiva ao utilizar sistema de custeio e controle de custos. In: CONGRESSO DE ADMINISTRAÇÃO HOSPITALAR, 2009, São Paulo: ADH, 2009.

LEAL, R. M; MATOS, J. B. B. Perfil etário de beneficiários de planos de saúde de assistência médica no Brasil: uma análise comparativa do mercado individual com o coletivo. In: CONGRESSO BRASILEIRO DE CIÊNCIAS SOCIAIS E HUMANAS EM SAÚDE; XIV CONGRESSO DA ASSOCIAÇÃO INTERNACIONAL DE POLÍTICA DE SAÚDE; X CONGRESSO DA ASSOCIAÇÃO LATINO-AMERICANA DE MEDICINA SOCIAL, 4, 2007,
Salvador: ABRASCO, 2007. Disponível em: http://www.congressosalvador2007.com.br/trabalhos/trabalhos.php. Acesso em 01.07.2009.

LEAL, R. M; MATOS, J. B. B. Planos de saúde de assistência médica no Brasil: uma avaliação da regulação econômica dos produtos. Revista de Politicas Públicas e Gestão Governamental, v. 6, p. 79-104, 2008a. Disponível em: http://www.anesp.org.br/?q=node/1522. Acesso em 28.04.2008.

LEAL, R. M; MATOS, J. B. B. Planos de saúde no Brasil no período pós-regulação: uma análise do perfil de beneficiários e dos aspectos econômicos segundo tipo de contratação. II Prêmio de Saúde Coletiva do Instituto de Estudos em Saúde Coletiva da Universidade Federal do Rio de Janeiro, 2008b. Disponível em: www.iesc.ufrj.br/cursos/semanadesaudecoletiva/ result_Premio.html. Acesso em 28.04.2008.

LIMA, C. R. M; LIMA, C. R. M. A avaliação do custo-eficácia das intervenções em organizações de saúde. RAE-revista de administração de empresas, v. 38, n. 2 , p. $62-73,1998$

MALIK, A. M. A questão da saúde no Brasil. RAE-revista de administração de empresas, v. 35, n. 4, 1995.

MEDICI, A. C; MARQUES, R. M. Sistemas de custo como instrumento de eficiência e qualidade dos serviços de saúde. Caderno Fundap, n.19, p. $47-59,1996$

NEVES, M. A. B. Estratégias de competição em saúde para o sistema único de saúde: uma proposta baseada nas ideias de Porter e Teisberg. Disponível em: http://www.seplag.rs.gov.br/uploads/Painel_44_Marco_Aurlio_Borges_formatado.pdf. Acesso em 01.07.2009.

OCKÉ-REIS, C. O; CARDOSO, S. S. Uma descrição do comportamento dos preços dos planos de assistência à saúde, 2001-2005. T.D. IPEA n $1.232,2006$.

PORTER, M. E; Estratégia competitiva. São Paulo: Campus, 1980.

PORTER, M; TEISBERG, E. Repensando a saúde: estratégias para melhorar a qualidade e reduzir os custos. Porto Alegre: Bookman, 2007.

RAMALHO, P. I. S. (Org) Regulação e agências reguladoras: governança e análise de impacto regulatório. Brasília: ANVISA, 2009. Disponível em: http://www.regulacao.gov.br/publicacoes/livros/regulacao-e-agencias-reguladoras. Acesso em 01.07.2009.

SAINTIVE, M. B; CHACUR, R. S. A regulação tarifária e o comportamento dos preços administrados. Brasília: Seae/MF, Documento de trabalho, n. 33, 2006.

SANTOS, F. P. A regulação publica da saúde no Brasil: o caso da saúde complementar. Tese de Doutorado em Saúde Coletiva. 2006, Programa de PósGraduação da Faculdade de Ciências Médicas da Universidade Estadual de Campinas, Campinas, 2006.

TEIXEIRA, L. S. Reajustes de preços administrados no setor saúde. Estudo da Consultoria Legislativa da Câmara dos Deputados. Fevereiro de 2006. Disponível em: http://www2.camara.gov.br/internet/publicacoes/estnottec/ tema10/2005_7377.pdf. Acesso em 09.09.2007.

ZUCCHI, P; DEL NERO, C; MALIK, AM. Gastos em saúde: os fatores que agem na demanda e na oferta dos serviços de saúde. Saúde e Sociedade, n. 9, p. $27-150,2000$ 